\title{
Can atorvastatin with metformin change the natural history of prostate cancer as characterized by molecular, metabolomic, imaging and pathological variables? A randomized controlled trial protocol.
}

\author{
Matthew J. Roberts a, b, c, d_E: m.roberts2@uq.edu.au \\ John W Yaxley ${ }^{\text {b }}$ - E: dryaxley@wesleyurologyclinic.com.au \\ Geoffrey D Coughlin ${ }^{\text {b }}$ - E: geoff_coughlin@me.com
}

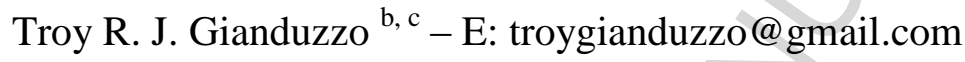

Rachel C. Esler ${ }^{\text {b }}$ - E: rachel.esler@gmail.com

Nigel T. Dunglison ${ }^{\mathrm{b}}$ - E: nigeldunglison@gmail.com

Suzanne K. Chambers ${ }^{\text {a,e }}$ - E: suzanne.chambers@ griffith.edu.au

Robyn J Medcraft ${ }^{\text {a, b }}$ - E: r.medcraft@uq.edu.au

Clement W. K. Chow a-E: c.chow3@uq.edu.au

Horst Joachim Schirra ${ }^{\text {d }}$-E: horst.schirra@cai.uq.edu.au

Renee S. Richards ${ }^{\text {a }}$ - E: r.richards@uq.edu.au

Nicholas Kienzle ${ }^{\mathrm{f}}-$ E: nicholas.kienzle@health.qld.gov.au

Macy Lu ${ }^{\mathrm{f}}-$ E: macy.lu@ health.qld.gov.au

Ian Brereton ${ }^{\mathrm{d}}$ - E: i.brereton@uq.edu.au

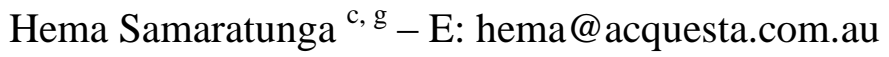

Joanna Perry-Keene $^{\mathrm{h}}$ - E: Joanna.Perry-Keene@ health.qld.gov.au

Diane Payton $^{\mathrm{h}}$ - E: Diane.Payton@health.qld.gov.au

Chikara Oyama ${ }^{\text {i }}$ - E: coyama@cc.hirosaki-u.ac.jp

Suhail A. Doi ${ }^{\mathrm{j}, \mathrm{k}, 1}$ - E: sardoi@gmx.net 
Martin F. Lavin ${ }^{\text {a }}$ - E: m.lavin@uq.edu.au

Robert A. Gardiner a, b, c, m \# - E: f.gardiner@uq.edu.au

${ }^{a}$ The University of Queensland, Centre for Clinical Research, Building 71/918, RBWH, Herston Brisbane, Qld, 4029, Australia

${ }^{\mathrm{b}}$ Department of Urology, Royal Brisbane and Women's Hospital, Brisbane, Qld, 4029, Australia

${ }^{\mathrm{c}}$ School of Medicine, The University of Queensland, Herston, Qld, 4006, Australia

${ }^{\mathrm{d}}$ The Centre for Advanced Imaging, The University of Queensland, St. Lucia, Brisbane, Qld 4072, Australia

${ }^{\mathrm{e}}$ Griffith Health Institute, Griffith University, Gold Coast, Qld 4222, Australia

${ }^{\mathrm{f}}$ Department of Medical Imaging, Royal Brisbane and Women's Hospital, Brisbane, Qld, 4029

${ }^{g}$ Aquesta Pathology, PO Box 1878, Toowong DC, Brisbane, Qld, 4066, Australia

${ }^{\text {h }}$ Anatomical Pathology, Pathology Queensland, Herston, Brisbane, Qld, 4029, Australia

${ }^{i}$ Department of Urology, Hirosaki University Graduate School of Medicine, 5-Zaifu-cho, Hirosaki 036-8562, Japan

${ }^{\mathrm{j}}$ Research School of Population Health, The Australian National University, Canberra, ACT, Australia

${ }^{\mathrm{k}}$ School of Agricultural, Computational and Environmental Sciences, University of Southern Queensland, Toowoomba, Australia

${ }^{1}$ College of Medicine, Qatar University, Doha, Qatar

${ }^{\mathrm{m}}$ Edith Cowan University, Joondalup, WA, 6027, Australia

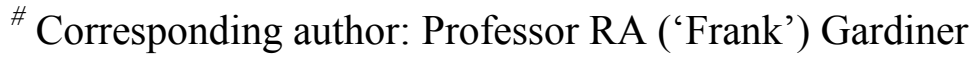

UQ Centre for Clinical Research (UQCCR) 
Level 6, Building 71/918 Royal Brisbane Hospital, Herston, QLD 4006

Phone : +61 733466046

Fax: +61 $733465509 \quad$ Email: f.gardiner@uq.edu.au 


\begin{abstract}
Background

Atorvastatin and metformin are known energy restricting mimetic agents that act synergistically to produce molecular and metabolic changes in advanced prostate cancer (PCa). This trial seeks to determine whether these drugs favourably alter selected parameters in men with clinicallylocalized, aggressive PCa.

\section{Methods/design}

This prospective phase II randomized, controlled window trial is recruiting men with clinically significant PCa, confirmed by biopsy following multiparametric MRI and intending to undergo radical prostatectomy. Ethical approval was granted by the Royal Brisbane and Women's Hospital Human and The University of Queensland Medical Research Ethics Committees.

Participants are being randomized into four groups: metformin with placebo; atorvastatin with placebo; metformin with atorvastatin; or placebo alone. Capsules are consumed for 8 weeks, a duration selected as the most appropriate period in which histological and biochemical changes may be observed while allowing prompt treatment with curative intent of clinically significant PCa. At recruitment and prior to RP, participants provide blood, urine and seminal fluid. A subset of participants will undergo 7Tesla magnetic resonance spectroscopy to compare metabolites in-vivo with those in seminal fluid and biopsied tissue.

The primary end point is biochemical progression, defined using biomarkers (serum prostate specific antigen; PCA3 and citrate in seminal fluid and prostatic tissue). Standard pathological assessment will be undertaken alongside quality of life and psychosocial outcome assessments. 


\section{Discussion}

This study is designed to assess the potential synergistic action of metformin and atorvastatin on

PCa tumour biology. The results may determine simple methods of tumour modulation to reduce disease progression.

\section{Keywords}

Prostate cancer; atorvastatin; metformin; clinical trial; biomarkers; metabolomics 


\section{INTRODUCTION}

Aggressive prostate cancer $(\mathbf{P C a})^{1}$ cells increase glucose uptake and glycolysis under normoxic conditions (the Warburg effect [1]) producing glycolytic intermediates that also feed biosynthesis and PCa proliferation. $[2,3]$. Metformin reduces glucose oxidation to increase glutamine metabolism and cell death while inhibiting metastatic behaviour. Epidemiological evidence suggests metformin use is associated with reduced risks of many cancers, including PCa $[4,5]$ with reduced hyperinsulinaemia by metformin in advanced $\mathrm{PCa}$ potentially improving androgen deprivation therapy (ADT) response [6]. When statins are combined with metformin, further reduction in PCa progression and improved clinical outcomes have been reported, indicating a potential additive or synergistic effect to this medication combination [7-9]. Statins reduce cholesterol and mevalonic acid biosynthesis, with in vitro evidence that statins slow testosterone synthesis by inhibiting pre-cursor molecule transport, improving ADT response [10]. Hypercholesterolaemia is associated with high risk $\mathrm{PCa}[11]$ and androgen-independent $\mathrm{PCa}$ metastasis [12] with statin use associated with lower prostate specific antigen (PSA) levels, percentage positive biopsies and fewer cases of advanced and fatal disease [8]. Lipophilic statins such as atorvastatin also inhibit

\footnotetext{
${ }^{1}$ Abbreviations: ADT - androgen deprivation therapy; bEvo - biochemical progression; DSS - 4,4-dimethyl-4-silapentane-1-sulfonic acid-d 6 ; DFTMP - difluorotrimethylsilanylphosphonic acid; ERMA - energy restriction mimetic agents; FID - free induction decay; GPC - glycerophosphocholine; ISUP - International Society of Urological Pathology; mpMRI - multiparametric magnetic resonance imaging; MRS - magnetic resonance spectroscopy; NMR - nuclear magnetic resonance; OPLS - orthogonal projections to latent structures; PBS - phosphate buffered saline; PCa - prostate cancer; PCA3 - prostate cancer antigen 3; PLS - partial least squares; PSA - prostate specific antigen; RBWH - Royal Brisbane and Women's Hospital; RP - radical prostatectomy; SF - seminal fluid, SF-36 -
} Short Form Health Survey 36 
PCa cell migration to bone marrow stroma [13], however benefit in reducing biochemical recurrence, remains uncertain [14].

Medication safety profiles of metformin and atorvastatin are favourable, with significant side effects rarely observed. Concern for metformin regarding lactic acidosis is reserved for patients with significant comorbidities (chronic renal failure, congestive cardiac failure) [15]. Large cohorts consuming statins report rhabdomyolysis in up to 11 per 100,000 person-years [16]. The use of metformin as a neoadjuvant agent for 4-12 weeks by Joshua and colleagues was well tolerated and demonstrated a $10 \%$ reduction in PSA, $6.5 \%$ reduction in IGF-1 and $5 \%$ reduction in BMI [17]. Such ideal drug tolerability and favourable clinical effects supports their adjunctive use in localized prostate cancer without need for a phase I controlled trial in this context.

Metformin and atorvastatin may influence malignant metabolic transformation in the prostate, known to favour ATP production and fatty acid synthesis, by shifting citrate, detectable in seminal fluid (SF) [18-20]. Markers, such as prostate cancer antigen 3 (PCA3), improve PCa detection and disease monitoring but may vary with epigenetic and exogenous stimuli [21, 22].

Initially promising findings by Joshua and colleagues demonstrated significant changes in molecular markers following neoadjuvant metformin therapy prior to RP [17]. These medications are also being explored in Metformin Active Surveillance Trial (MAST) Study (NCT01864096) in delaying pathologic disease progression. Thus, exploring the role of energy restriction mimetic agents (ERMAs) represents an exciting development in managing men with PCa. However, before atorvastatin and metformin can be entertained for use in 
patients with early $\mathrm{PCa}$, their potential demonstrable beneficial effects with respect to tumour parameters need to be evaluated objectively.

The primary aim of this study is to determine whether these drugs by themselves and together, favourably alter selected parameters in a group of clinically-localized, aggressive PCas. 


\section{MATERIALS AND METHODS}

\subsection{Study design}

This is a prospective randomized, double-blinded controlled phase II window trial designed to determine the efficacy on biochemical progression of atorvastatin and metformin, in isolation and together, in a population of men with early, clinically significant PCa. In addition, the effect of these drugs on PCa biology will be assessed in a population not previously studied in this respect whilst these men await definitive treatment by radical prostatectomy (RP), in accordance with a phase II window trial design [23]. Men with an elevated PSA who have a multiparametric magnetic resonance imaging (mpMRI) examination that demonstrates a PI-RADS 4 or 5 lesion and who, at consultation, express an intention to proceed to RP should biopsy confirm the suspicion of high-risk PCa, will be approached to enter the study. Our current practice includes in depth counselling prior to biopsy in order to ascertain the benefits to the patient in investigating for PCa. This includes outlining the biopsy and treatment process, with treatment options of surgery, radiotherapy, active surveillance or watchful waiting all discussed. Following written informed consent and randomisation by the manufacturing pharmacy (QPharm) to ensure clinician and participant blinding, four study groups are being examined, as outlined in Figure 1.

The protocol is designed and reported according to the SPIRIT guidelines [24]. Participants will provide blood, urine and SF after $48 \mathrm{hrs}$ abstinence of sexual activity. Blood and SF samples will be used to determine biomarkers of interest as defined by the primary and secondary endpoints. Further exploratory analyses will be conducted as outlined in order to determine biochemical effects of these medications in this patient cohort. Prior to giving specimens, a subset of participants, selected by a sub-randomisation process, will opportunistically undergo a further mpMRI with MRS using a 7 Tesla machine at the University of Queensland Centre for Advanced 
Imaging. Here, we will assess the metabolic profile of participants prostates in-vivo, for comparison with those seen in seminal fluid in vitro, and ascertain if superior imaging is provided by this machine. Participants will then undergo transperineal prostate biopsy targeting lesions of interest (cognitive biopsies) detected by pre-trial mpMRI, in addition to systematic whole gland biopsies using a template as per the local department protocol. Biopsy samples from index lesions and from non-index lesion areas will be taken for research purposes and stored for subsequent molecular and nuclear magnetic resonance (NMR) analysis.

Participants will undergo 8 weeks of capsule consumption, as this duration was determined to be most appropriate in which histological and biochemical changes may be observed while allowing prompt treatment with curative intent of clinically significant PCa. Non-invasively obtained participant samples will be collected again and mpMRI with magnetic resonance spectroscopy (MRS) will be repeated (for those previously randomized to have these investigations). The reason for allocating only a limited number of participants for mpMRI and MRS with the 7 Tesla machine is cost. The biomarker kinetic changes following biopsy are poorly described, however we expect these will be minimally affected by biopsy artefacts with 8 weeks of treatment and healing. Latifoltojar and colleagues examined changes in mpMRI parameters following biopsy and described a return to baseline apparent diffusion coefficient (ADC) parameters 1 month post biopsy [25]. The effects of biopsy on MRS parameters are currently unknown and will be examined in this study. 


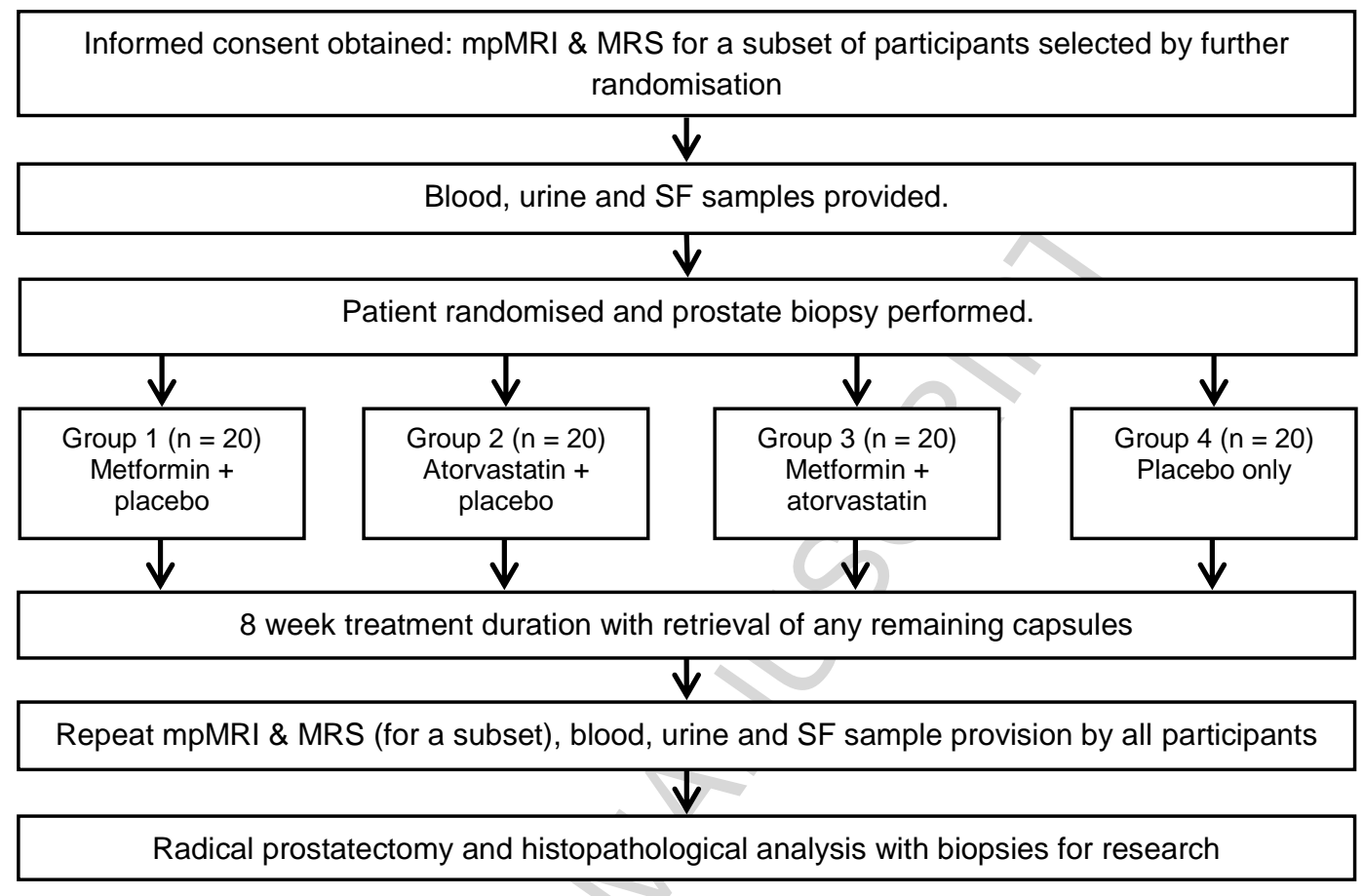

Figure 1: Flow chart of study design, incorporating timing of specimen collection, imaging and capsule distribution.

Ethical approval has been obtained from the Royal Brisbane and Women's Hospital (RBWH)

Human Research Ethics Committee (Approval no. HREC/14/QRBW/153 together with

HREC/09/QRBW/320, HREC/09/QRBW/305 and 1995/088B) and The University of

Queensland Medical Research Ethics Committee (Approval no. 2014000944 together with 2006000262) using the National Ethical Application Form. Specialist clinicians are overseeing all aspects of management through our established team. This trial has been registered in the Australian New Zealand Clinical Trials Registry (Registration number:

ACTRN12615000571572).

Consent to participate in the trial is being obtained from treating urologists or the Trial Coordinator (RM). This process also includes provision for storage and future use of clinical data and biological specimens. Database access is password-protected and limited to the Trial Coordinator (RM) who will then provide information as necessary to the other investigators. The 
database will be stored on a secure internal (UQ) server. At the conclusion of the trial, data access will be limited to the Trial Coordinator (RM) and relevant Principal Investigators (MJR, RAG).

Adverse events are being monitored by the Trial Coordinator (RM), who contacts participants by telephone on two occasions during the trial to ensure satisfaction and determine the presence of any medication side-effects, or other difficulties. In addition, participants are instructed to contact the trial coordinator should they have any concerns or difficulties at other times during the trial. Further reports to the participants treating urologists and general practitioners, as well as relevant hospitals will be included and promptly assessed in detail.

Untoward events are being determined by participant experiences of new symptoms during the study period, as well as routine monitoring using pathology tests, and the results of any other investigations prompted by other health professionals (e.g. general practitioners, emergency department clinicians) during the study period. The Clavien system is being used to document any adverse effects reported, while study questionnaires are also being monitored for participant satisfaction. Adverse outcomes and trial conduct are regularly audited and discussed with the relevant ethics representatives.

The funding source had no involvement in study design; in the collection, analysis and interpretation of data; in the writing of the report; and in the decision to submit the article for publication.

\subsection{Study population and Recruitment}

Participants are being recruited from the RBWH Urology Outpatient clinic and specialist private practices in Brisbane, Queensland. Clinicians identify men as having clinically significant PCa 
on the basis of prostate imaging with mpMRI and who intend to proceed to RP. Following informed consent, those fulfilling all inclusion criteria and no exclusion criteria are entered into the randomization process. Recruitment is promoted by treating urologists discussing the study with eligible participants.

\section{$\underline{\text { Inclusion criteria }}$}

- $\quad$ suspected of having high-risk PCa on the basis of mpMRI (PIRADS 4/5 lesions), with clinically-localized PCa and who intend to proceed to RP following confirmation of their disease as high-risk following biopsies;

- able to provide informed consent (written and verbal) in English;

- considered by their urologist that involvement in the research programme/ involvement in the study is considered to be their best interests, for appropriate selection of patients who are likely to benefit from treatment;

- $\quad$ prepared and able to provide a specimen of SF for monitoring before biopsy and at the completion of treatment, just before RP;

- willing to provide urine and serum samples prior to biopsy and again before RP

- able to lie flat and willing to undergo mpMRI/MRS scanning on two occasions during the trial period with no previous or current history of claustrophobia;

- normal fasting blood glucose, kidney and liver function tests;

\section{$\underline{\text { Exclusion criteria }}$}

- previous history of head injury, dementia, psychiatric illness or concurrent cancer;

- regular administration of any lipid-lowering medication or blood-glucose lowering drugs

- prior experience of any adverse effects with lipid or glucose-lowering drugs; 
- currently taking drugs known or thought to have an interaction with metformin and atorvastatin;

- previous ingestion of a 5-alpha reductase inhibitor drug;

- known co-morbidities that would contraindicate commencement of metformin or atorvastatin, such as chronic kidney disease, congestive heart failure, liver disease.

\subsection{Randomisation and allocation concealment}

Following enrolment, participants are randomized using a centralized database by an external research pharmaceutical organisation (QPharm Pty Ltd), which is experienced in participation and formulation of drugs for its own and other clinical trials. The randomization is stored and concealed by QPharm, resulting in blinding of clinicians and participants to study medications. Unblinding will be permissible in the event of an adverse event resulting in cessation of medication use, with liaison between the investigators, QPharm and the participant. All medications are identical in appearance to maintain blinding during medication ingestion.

\subsection{Intervention}

Participants are randomized into one of the four study groups:

1) metformin $500 \mathrm{mg}$ twice a day with placebo once a day;

2) atorvastatin $20 \mathrm{mg}$ once a day with placebo twice a day;

3) metformin $500 \mathrm{mg}$ twice a day with atorvastatin $20 \mathrm{mg}$ once a day;

4) placebo three times a day.

All capsules have been formulated by QPharm Pty Ltd to appear identical in order to comply with blinding for participants and investigators.

\subsection{Outcome measures}




\section{$\underline{\text { Primary endpoint }}$}

The primary end point is serum PSA "biochemical evolution" (bEvo), defined as an increase inSerum PSA prior to surgery and following 8 weeks of treatment from baseline measurement of $20 \%$ (based on values of reported studies $[\mathbf{1 7}, \mathbf{2 6}, \mathbf{2 7}]$ ). These studies were used instead of those from larger observational studies [28, 29] as the observational studies had a low (25-30\%) proportion of clinically significant PCa. The term "biochemical progression" is intended for this specific definition and different from "biochemical recurrence" commonly used in the posttreatment scenario.

\section{$\underline{\text { Secondary endpoints }}$}

Secondary endpoints that are being assessed opportunistically are biochemical progression for seminal fluid-based biomarkers PCA3:PSA and citrate, defined for each biomarkers as follows:

a) PCA3:PSA measured in seminal fluid: Increase from baseline measurement by $20 \%$ (based on improved all-cause and cancer-specific survival estimates[30]).

b) Citrate measured in seminal fluid and prostatic biopsies at the time of transperineal biopsies and the prostate ex-vivo after RP as well as MRS: Increase from baseline measurement of $30 \%$ (based on serum citrate association with aggressive prostate cancer [19]).

These biochemical markers are being preferentially assessed ahead of tissue histological markers on the basis that biochemical effects precede histological change, known as the 'field effect' in cancer biology [31], and are thus more likely to be detected in vitro using seminal fluid and in vivo using MRSI. 


\section{$\underline{\text { Clinical Data Collection }}$}

Clinical data will be obtained by participating urologists and the Trial Coordinator (RM), who contacts participants by telephone on at least two occasions during the trial to ensure participant satisfaction and determine the presence of any side-effects or other difficulties. The Trial Coordinator is experienced in the data collection tools being used. Data will be collected onto a CAISIS database, used in our randomized, controlled study of open and robotic prostatectomy $[32,33]$ and with which we are familiar.

\section{Assessment and Follow-up}

Participants are being assessed for inclusion upon enrolment for prostate biopsy. Those whose biopsies do not include any Gleason 4 or 5 tumour do not receive drug and do not take any further part in the study. Participants continuing receive medication, as per randomization protocol, and are placed on the waiting list for surgery which is planned for 8 weeks after their biopsies.

Serum investigations used clinically include total PSA (Abbott Architect ${ }^{\circledR}$ assay) and free-total PSA estimations as well as serum testosterone and metabolic screening (serum biochemical and lipoprotein profiles). Blood tests will all be performed according to standard assays by Sullivan \& Nicolaides Pathology in addition to glycoslylated PSA quantified as previously described [34].

The intended duration from diagnosis and treatment is 8 weeks, with assessments performed prior to biopsies and again just before RP. mpMRI and MRS will be assessed by IB, NK and ML. Biopsy and RP slides will be reviewed by specialized uropathologists (JP-K, JDP, MLTHS) with reference to the 2014 International Society of Urological Pathology (ISUP) classification [35] for continuity and expert interpretation. Standard parameters for biopsies (total number of 
cores, number and percentage of positive cores, Gleason/ISUP score) and RP specimens (gland size, margin status, extracapsular status, pathological stage, Gleason score) are being reported. Surgical follow-up is being determined by the treating urologist with follow-up assessments performed at these appointments until 24 months post-operatively.

Technical details regarding biological sampling and storage as well as molecular and metabolomic investigations are outlined in the Supplementary Material.

\subsection{Sample size and statistical analysis}

Sample sizes were based on a randomized selection design with the aim of achieving a $80 \%$ probability that the best schedule produced the highest observed response rate [36-38]. We took the expected baseline freedom from biochemical progression rate for the placebo schedule to be $80 \%$ based on results reported for the serum PSA biomarker [27]. We estimate that we need to study 20 participants per schedule, to have a $80 \%$ probability of selecting the schedule that has a true freedom from biochemical progression rate that is at least 15\% higher assuming this is the minimal practically significant difference. This calculation can be verified through the online calculator from the Center for Clinical Research and Biostatistics of the Chinese University of Hong Kong (https://www2.ccrb.cuhk.edu.hk/stat/phase2/Randomized.htm). We were unable to suitably estimate the proportion of men who would be enrolled with a negative biopsy result and so are continuing to recruit until the sample size is achieved.

Differences in categorical variables between groups will be tested using chi squared analysis while continuous variables will be compared using two-sample $t$ tests. Univariate analysis will be performed using the binary bEvo variable as the outcome and intervention schedule category as the predictor using a GLM with a Poisson family, log link and robust error variances in order to 
generate the relative risk. Multivariable analysis will also be performed using a similar GLM to determine the independence of the schedule of treatment from other relevant explanatory variables. The latter will be included in the multivariable model if they are found to be associated with outcome $(\mathrm{P}<0.2)$ on univariate analysis. Link specification will be tested using the squared linear predictor while the goodness of fit of the model will be evaluated by the ability of the linear predictor from the model to classify participants into progressors and non-progressors (i.e., its predictive performance) will be evaluated using the $\mathrm{C}$ statistic, a term equivalent to the area under a receiver operating characteristic curve for the dichotomous outcome (bEvo). All analysis will be done using Stata (StataCorp, College Station, TX) and $\mathrm{P}<0.05$ will be the threshold for significance.

\section{DISCUSSION}

Since commencing the study in March 2015, 9 participants have been recruited and 2 have completed the treatment and assessment protocols. One man with a mpMRI PI-RADS 4 lesion did not have PCa detected with his biopsies. As a consequence his participation in the trial was terminated. Compared with recruitment for altruistic SF donation by patients without likelihood of personal benefit, recruitment for this study, which includes SF donation as an essential requisite, has been much easier but considerable greater difficulty is being experienced identifying men who have not been consuming a statin regularly. 


\section{Competing interests}

All authors declare no financial or personal conflicts of interest. This research received financial support from Royal Brisbane and Women's Hospital Foundation. MJR is supported by a Doctor in Training Research Scholarship from Avant Mutual Group Ltd, Cancer Council Queensland PhD Scholarship and Professor William Burnett Research Fellowship from the Discipline of Surgery, School of Medicine, The University of Queensland.

\section{Authors' contributions}

Drafting the study protocol: MJR, RM, RAG; Manuscript design and initial drafting: MJR, RAG; Manuscript Revision: MJR, TRJG, SK, HJS, RSR, SAD, RAG; Trial co-ordination: RM; Trial recruitment: MJR, JWY, GDC, TRJG, NTD, RCE, RAG; Imaging co-ordination and interpretation: NK, IB, ML; Pathology co-ordination and interpretation: HS, JP-K, DP; Laboratory and other data design and coordination: MJR, SK, CC, RR, HJS, SAD, MFL, RAG; All authors have read and approved the final manuscript.

\section{Acknowledgements}

None 


\section{References}

[1] Warburg O. On the origin of cancer cells. Science 1956;123(3191):309-14.

[2] Vander Heiden MG, Cantley LC, Thompson CB. Understanding the Warburg effect: the metabolic requirements of cell proliferation. Science 2009;324(5930):1029-33.

[3] Schulze A, Harris AL. How cancer metabolism is tuned for proliferation and vulnerable to disruption. Nature 2012;491(7424):364-73.

[4] Brown KA, Samarajeewa NU, Simpson ER. Endocrine-related cancers and the role of AMPK. Mol Cell Endocrinol 2013;366(2):170-9.

[5] Pollack MN. Insulin, insulin-like growth factors, insulin resistance, and neoplasia. The Am J Clin Nutr 2007;86(3):s820-2.

[6] Clements A, Gao B, Yeap SH, et al. Metformin in prostate cancer: two for the price of one. Ann Oncol 2011;22(12):2556-60.

[7] Danzig MR, Kotamarti S, Ghandour RA, et al. Synergism between metformin and statins in modifying the risk of biochemical recurrence following radical prostatectomy in men with diabetes. Prostate Cancer Prostatic Dis 2015;18(1):63-8.

[8] Moon H, Hill MM, Roberts MJ, et al. Statins: protectors or pretenders in prostate cancer? Trends Endocrinol Metab 2014;25(4):188-96.

[9] Chen-Pin W, Javier H, Lorenzo C, et al. Statins and Finasteride Use Differentially Modify the Impact of Metformin on Prostate Cancer Incidence in Men with Type 2 Diabetes. Ann Transl Med Epidemiol 2014;1(1).

[10] Harshman LC, Wang X, Nakabayashi M, et al. Statin Use at the Time of Initiation of Androgen Deprivation Therapy and Time to Progression in Patients With HormoneSensitive Prostate Cancer. JAMA Oncol 2015;1(4):495-504.

[11] Platz EA, Clinton SK, Giovannucci E. Association between plasma cholesterol and prostate cancer in the PSA era. Int J Cancer 2008;123(7):1693-8.

[12] Moon H, Ruelcke JE, Choi E, et al. Diet-induced hypercholesterolemia promotes androgenindependent prostate cancer metastasis via IQGAP1 and caveolin-1. Oncotarget 2015;6(10):7438-53.

[13] Brown M, Roulson JA, Hart CA, et al. Arachidonic acid induction of Rho-mediated transendothelial migration in prostate cancer. Br J Cancer 2014;110(8):2099-108.

[14] Scosyrev E, Tobis S, Donsky H, et al. Statin use and the risk of biochemical recurrence of prostate cancer after definitive local therapy: a meta-analysis of eight cohort studies. $B J U$ Int 2013;111(3 Pt B):E71-7.

[15] Bolen S, Feldman L, Vassy J, et al. Systematic Review: Comparative Effectiveness and Safety of Oral Medications for Type 2 Diabetes Mellitus. Ann Intern Med 2007;147(6):386-99.

[16] Law M, Rudnicka AR. Statin Safety: A Systematic Review. Am J Cardiol 2006;97(8, Supplement 1):S52-S60.

[17] Joshua, A.M., et al., A pilot 'window of opportunity' neoadjuvant study of metformin in localised prostate cancer. Prostate Cancer Prostatic Dis 2014;17(3): 252-8.

[18] Roberts MJ, Richards RS, Gardiner RA, et al. Seminal fluid: a useful source of prostate cancer biomarkers? Biomark Med 2015;9(2):77-80.

[19] Mondul AM, Moore SC, Weinstein SJ, et al. Metabolomic analysis of prostate cancer risk in a prospective cohort: The alpha-tocolpherol, beta-carotene cancer prevention (ATBC) study. Int J Cancer 2015;137(9):2124-32. 
[20] Roberts MJ, Schirra HJ, Lavin MF, et al. Metabolomics: a novel approach to early and noninvasive prostate cancer detection. Korean J Urol 2011;52(2):79-89.

[21] Roberts MJ, Chow CW, Schirra HJ, et al. Diagnostic performance of expression of PCA3, Hepsin and miR biomarkers inejaculate in combination with serum PSA for the detection of prostate cancer. Prostate 2015;75(5):539-49.

[22] Roberts MJ, Richards RS, Chow CW, et al. Prostate-based biofluids for the detection of prostate cancer: a comparative study of the diagnostic performance of cell-sourced RNA biomarkers. Prostate Int 2016 (in press).

[23] Glimelius, B. and M. Lahn, Window-of-opportunity trials to evaluate clinical activity of new molecular entities in oncology. Ann Oncol 2011;22(8):1717-25.

[24] Chan AW, Tetzlaff JM, Altman DG, et al. SPIRIT 2013 statement: defining standard protocol items for clinical trials. Ann Intern Med 2013;158(3):200-7.

[25] Latifoltojar, A., et al., Evolution of multi-parametric MRI quantitative parameters following transrectal ultrasound-guided biopsy of the prostate. Prostate Cancer Prostatic Dis 2015;18(4):343-51.

[26] Ku JH, Jeong CW, Park YH, et al. Relationship of statins to clinical presentation and biochemical outcomes after radical prostatectomy in Korean patients. Prostate Cancer Prostatic Dis 2011;14(1):63-68.

[27] Gutt R, Tonlaar N, Kunnavakkam R, et al. Statin Use and Risk of Prostate Cancer Recurrence in Men Treated With Radiation Therapy. J Clin Oncol 2010;28(16):2653-59.

[28]Wilt, T.J., et al., Radical Prostatectomy versus Observation for Localized Prostate Cancer. $N$ Engl J Med 2012;367(3): 203-213.

[29] Bill-Axelson, A., et al., Radical prostatectomy versus watchful waiting in early prostate cancer. N Engl J Med 2011;364(18):1708-17.

[30] Zhong S, Zhang X, Chen L, et al. Statin use and mortality in cancer patients: Systematic review and meta-analysis of observational studies. Cancer Treat Rev 2015;41(6):554-67.

[31] Costello, L.C. and R.B. Franklin, Prostatic fluid electrolyte composition for the screening of prostate cancer: a potential solution to a major problem. Prostate Cancer Prostatic Dis 2009;12(1):17-24.

[32] Gardiner RA, Yaxley J, Coughlin G, et al. A randomised trial of robotic and open prostatectomy in men with localised prostate cancer. BMC Cancer 2012;12:189.

[33] Gardiner RA, Coughlin GD, Yaxley JW, et al. A progress report on a prospective randomised trial of open and robotic prostatectomy. Eur Urol 2014;65(3):512-5.

[34] Ishibashi Y, Tobisawa Y, Hatakeyama S, et al. Serum tri- and tetra-antennary N-glycan is a potential predictive biomarker for castration-resistant prostate cancer. Prostate 2014;74(15):1521-9.

[35] Epstein JI, Egevad L, Amin MB, et al. The 2014 International Society of Urological Pathology (ISUP) Consensus Conference on Gleason Grading of Prostatic Carcinoma: Definition of Grading Patterns and Proposal for a New Grading System. Am J Surg Pathol 2015.

[36] Simon R, Wittes RE, Ellenberg SS. Randomized phase II clinical trials. Cancer Treat Rep 1985;69(12):1375-81.

[37] Mandrekar SJ, Sargent DJ. Randomized phase II trials: time for a new era in clinical trial design. J Thorac Oncol 2010;5(7):932.

[38] Rubinstein L, Crowley J, Ivy P, et al. Randomized phase II designs. Clin Cancer Res 2009;15(6):1883-90. 


\section{Accepted Manuscript}

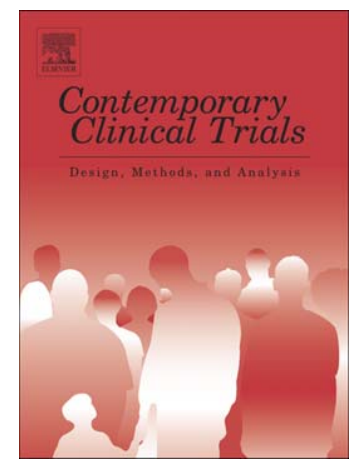

Matthew J. Roberts, John W. Yaxley, Geoffrey D. Coughlin, Troy R.J. Gianduzzo, Rachel C. Esler, Nigel T. Dunglison, Suzanne K. Chambers, Robyn J. Medcraft, Clement W.K. Chow, Horst Joachim Schirra, Renee S. Richards, Nicholas Kienzle, Macy Lu, Ian Brereton, Hema Samaratunga, Joanna Perry-Keene, Diane Payton, Chikara Oyama, Suhail A. Doi, Martin F. Lavin, Robert A. Gardiner

PII:

S1551-7144(16)30096-9

DOI: doi: 10.1016/j.cct.2016.06.014

Reference: CONCLI 1413

To appear in: $\quad$ Contemporary Clinical Trials

Received date: 23 December 2015

Revised date: $\quad 22$ June 2016

Accepted date: 26 June 2016

Please cite this article as: Roberts Matthew J., Yaxley John W., Coughlin Geoffrey D., Gianduzzo Troy R.J., Esler Rachel C., Dunglison Nigel T., Chambers Suzanne K., Medcraft Robyn J., Chow Clement W.K., Schirra Horst Joachim, Richards Renee S., Kienzle Nicholas, Lu Macy, Brereton Ian, Samaratunga Hema, Perry-Keene Joanna, Payton Diane, Oyama Chikara, Doi Suhail A., Lavin Martin F., Gardiner Robert A., Can atorvastatin with metformin change the natural history of prostate cancer as characterized by molecular, metabolomic, imaging and pathological variables? A randomized controlled trial protocol, Contemporary Clinical Trials (2016), doi: 10.1016/j.cct.2016.06.014

This is a PDF file of an unedited manuscript that has been accepted for publication. As a service to our customers we are providing this early version of the manuscript. The manuscript will undergo copyediting, typesetting, and review of the resulting proof before it is published in its final form. Please note that during the production process errors may be discovered which could affect the content, and all legal disclaimers that apply to the journal pertain. 